\title{
Kinesin family member 20B regulates tongue cancer progression by promoting cell proliferation
}

\author{
ZHANG-YI LI, ZHI-XING WANG and CHANG-CHUN LI \\ Department of Stomatology, The Fifth Central Hospital of Tianjin, \\ Tianjin Medical University, Tanggu, Tianjin 300450, P.R. China
}

Received March 14, 2018; Accepted November 12, 2018

DOI: $10.3892 / \mathrm{mmr} .2019 .9851$

\begin{abstract}
Oral cancer refers to the malignant tumors that occur in the oral cavity, of which $80 \%$ are squamous cell carcinomas. The incidence of oral cancer accounts for $\sim 5 \%$ of the incidence of systemic malignancies, with rapid progression, extensive infiltration and poor prognosis. In the present study, Kinesin family member (KIF)20B, a member of Kinesin- 6 family, was identified as a potential biomarker which could promote cancer progression. A total of 82 patients were recruited and KIF20B expression levels were investigated by immunohistochemistry, and were divided into high and low groups based on the median of KIF20B expression levels. The clinicopathological features and survival-associated data of the two groups were analyzed and the results were provided as a table and by a Kaplan-Meier plot, respectively. Additionally, KIF20B was successfully silenced in two tongue cancer cell lines, CAL-27 and TCA-8113. MTT and colony formation assay were performed to determine the changes of cell proliferation in knocked down-KIF20B cell lines. In addition, proliferation-associated proteins Ki67 and PCNA were investigated, by western blotting. In animal experiments, subcutaneous tumor formation was performed with control cells and cells with knocked down KIF20B, to determine the inhibitory effect of KIF20B in vivo. Firstly, it was found that there was significantly high expression levels of KIF20B in tongue cancer patients $(\mathrm{P}<0.05)$. Patients with high expression of KIF20B had poorer clinicopathological results including tumor differentiation level, lymph node metastasis and clinical stages. The overall survival and relapse-free survival of high-expression group were also poor. Secondly, after successful establishment of cells with knocked down KIF20B, this resulted in a notable reduction in cell proliferation in vitro. Subsequent western blotting further confirmed that Ki67 and
\end{abstract}

Correspondence to: Dr Chang-Chun Li, Department of Stomatology, The Fifth Central Hospital of Tianjin, Tianjin Medical University, 41 Zhejiang Road, Tanggu, Tianjin 300450, P.R. China E-mail: wzxyfk_8030@sina.com

Key words: kinesin family member 20B, oral squamous cell carcinoma, proliferation
PCNA expression levels had a significant decline. Finally, it was demonstrated that knocking down KIF20B could inhibit tumor volume growth in vivo. In conclusion, the high level of KIF20B in oral squamous cell carcinoma was significantly associated with poor clinicopathological features and survival. KIF20B might promote cancer development through enhancing cell proliferation in vitro, and might be a potential biomarker of oral squamous cell carcinoma.

\section{Introduction}

Oral cancer refers to the malignant tumors that occur in the oral cavity, of which $80 \%$ are squamous cell carcinomas including tongue, buccal, gingival, palatal, lip, maxillary and mandibular, mouth and oropharyngeal cancers, salivary gland adenocarcinoma and maxillary sinus cancer, as well as cancers that occur in the facial skin $(1,2)$. The incidence of oral cancer accounts for $\sim 5 \%$ of the incidence of systemic malignancies (1,2). Overall, 49,670 oral cavity and pharynx cancer cases were estimated in 2017 in United States, which accounts for $\sim 3 \%$ of the incidence of systemic malignancies (2). Oral cancer has the characteristics of rapid progression, extensive invasion and poor prognosis (1). In early oral cancer, if there is no cervical lymph node metastasis, the use of surgery alone or radiation therapy has a good effect (1). In advanced oral cancer, surgical treatment combined with postoperative radiotherapy would be more suitable (1). However, the deficiency is that our understanding of oral cancer is not fully understood. The complexity of oral cancer made it hard to achieve significant results in the biological treatment of oral cancer. Therefore, biomarkers for oral cancer have not yet been fully investigated.

Kinesin superfamily proteins (KIFs) serve important roles in intracellular transport system and are essential for cellular morphology (3-12). KIFs are motors for transport of mitochondria during cell division (13), and function as a biomacromolecule transporter, such as protein complexes and mRNAs to microtubules and hydrolyzing adenosine triphosphatase for energy (14-16). These proteins are also involved in chromosome condensation and are essential in maintaining chromosomal integrity during mitosis (17-23). Kinesin-6 superfamily is a sub-family of KIFs, which function during cytokinesis, establishing telophase spindle structure and mobilizing mitotic regulator proteins $(24,25)$. Kinesin- 6 are also involved in microtubule transport (26). KIF20B, which is 
also known as membrane palmitoylated protein 1 (MPP-1) or MPHOSPH1, is a member of the Kinesin-6 family (3,4). KIF20B was first identified as a plus-end-directed kinesin-associated protein and was phosphorylated at the $\mathrm{G} 2 / \mathrm{M}$ transition $(3,4)$. KIF20B could also influence microtubule-binding, microtubule-bundling properties and microtubules-stimulated adenosine triphosphatase activity in vitro, and is essential for cytokinesis in cell cycle (5). KIF20B was recently found to serve important roles in multiple types of cancer, which could function as cancer-testis antigen specific to human bladder cancer $(4,6)$, and KIF20B was associated with cell proliferation, apoptosis and tumor growth in hepatocellular carcinoma targeting p53 (7). Several studies have indicated that KIF20B serves a role in tumor progression, including colorectal cancer, hepatocellular carcinoma, pancreatic cancer and bladder cancer (4,6-10). In addition, KIF20B predicts poor prognosis, and is associated with cell proliferation, apoptosis and tumor growth in hepatocellular carcinoma through targeting p53 $(7,10)$. However, the function and mechanism of KIF20B in different tumors has not been fully elucidated, and further discussions are still needed.

To explore the function and the role of KIF20B in tongue cancer, a total of 82 patients were recruited and KIF20B expression levels were investigated by immunohistochemistry. Next, the clinicopathological features and survival-associated data of the two groups were analyzed and the results were provided as a table and by a Kaplan-Meier plot, respectively. In addition, the function of KIF20B in tongue cancer in vitro and in vivo was examined. Therefore, through the above results, KIF20B might be a potential biomarker for tongue cancer and could become a novel target for biotherapy.

\section{Materials and methods}

Specimen collection. A total of 82 surgical tongue cancer specimens from January 2005 to March 2009 were collected from the Fifth Central Hospital of Tianjin. Age, sex, tumor stage, and pathologic diagnosis including lymphatic and venous invasion data were retrospectively collected. Participants provided written informed consent, whose preoperative diagnosis was clear, without other systemic serious diseases, with indications of surgical resection, with clear pathological diagnosis following surgical resection and with no serious systemic complications following surgery. No evidence of tumor metastasis was confirmed by cross-sectional imaging in all patients. The present study was approved by the Ethics Committee of the Fifth Central Hospital of Tianjin.

Immunohistochemistry (IHC) and scoring. IHC staining was performed on paraffin-embedded tissue sections $(4 \mu \mathrm{m}$ thickness) by using the polymer peroxidase method, with tongue cancer and adjacent non-cancerous samples fixed overnight in $20 \%$ phosphate buffered formalin $(\mathrm{pH} \mathrm{7.4)} \mathrm{at}$ room temperature. After deparaffinization with xylene and rehydration with an alcohol-water mixture, the sections were treated with $0.3 \%$ hydrogen peroxide in methanol at room temperature for $30 \mathrm{~min}$ before being heat-treated with $10 \mathrm{mM}$ citric acid ( $\mathrm{pH}$ 6.0) for antigen retrieval and in order to disrupt endogenous peroxidase activity. After washing with PBS, the sections were incubated with rabbit polyclonal anti-KIF20B antibody (cat. no. NBP1-88042; 1:100; Novus Biologicals, Ltd., Cambridge, UK) at $4^{\circ} \mathrm{C}$ overnight. After washing with PBS, slides were treated with horseradish peroxidase-conjugated goat anti-rabbit secondary antibody (cat. no. PV-6001; $75 \mu \mathrm{l}$; Zhongshan Jinqiao Biotechnology Co., Ltd., Beijing, China) for $30 \mathrm{~min}$ at room temperature. Staining with diaminobenzidine (cat. no. ZLI-9018; OriGene Technologies, Inc., Beijing, China), and then stained with $0.5 \%$ hematoxylin for $20 \mathrm{sec}$ at room temperature.

KIF20B expression scoring was based on the staining area and staining intensity. Semi-quantitative results were obtained to judge the percentage of microscopically positive cells and staining intensity. Five high-power fields (magnification, $\mathrm{x} 200$ ) were observed per slice, the percentage of positive cells was counted as follows: If the number of positive cells was $<5 \%$ this corresponded to 0 point, if it was $5-25 \%$ this corresponded to 1 point, if it was $26-50 \%$ this corresponded to 2 points, if it was $51-75 \%$ this corresponded to 3 points, and if it was $76-100 \%$ this corresponded to 4 points. Positive staining intensity: 0 for no color, 1 for light yellow, 2 for brown and 3 for dark brown. The two scores were multiplied by the positive rating: 0 was negative (-), 1-4 was weakly positive (+), 5-8 was positive $(++)$, and 9-12 was strongly positive $(+++)$.

Cell culture. CAL-27 and TCA8113 tongue cancer cell lines used in the present study, were obtained from the American Type Culture Collection (Manassas, VA, USA). CAL-27 was cultured in Dulbecco's Modified Eagle's Medium (DMEM; Gibco; Thermo Fisher Scientific, Inc., Waltham, MA, USA) with $10 \%$ fetal bovine serum and TCA8113 cells were cultured in RPMI-1640 (both Gibco; Thermo Fisher Scientific, Inc.) with $20 \%$ fetal bovine serum. The cells were kept in a $37^{\circ} \mathrm{C}$ incubator with $5 \% \mathrm{CO}_{2}$ and $40-70 \%$ humidity. Cells were regularly monitored using a light microscope, and subcultured as soon as they reached $80-90 \%$ confluency.

Reverse transcription-quantitative polymerase chain reaction $(R T-q P C R)$ and primers. Total RNA was extracted from cells using TRIzol reagent (Thermo Fisher Scientific, Inc.) according to the manufacturer's protocol. cDNA was synthesized from $2 \mu \mathrm{g}$ of total RNA with a random hexamer using the Roche-Transcriptor First Strand cDNA Synthesis kit (Roche Diagnostics, Basel, Switzerland). These cDNAs were used for the measurement of gene expression. The expression of KIF20B was quantified using RT-qPCR using the SYBR Green PCR kit (Applied Biosystems; Thermo Fisher Scientific, Inc.) The following thermocycling conditions were used: $95^{\circ} \mathrm{C}$ for $2 \mathrm{~min}$, followed by 35 cycles of $95^{\circ} \mathrm{C}$ for $5 \mathrm{sec}, 55^{\circ} \mathrm{C}$ for $30 \mathrm{sec}$ and $72^{\circ} \mathrm{C}$ for $30 \mathrm{sec}$, and final extension at $72^{\circ} \mathrm{C}$ for $6 \mathrm{~min}$. GAPDH was used as the internal reference gene. The primers sequences were: KIF20B forward, 5'-CCGGGAAAG TAAACTGACTCAC-3' and reverse, 5'-TTCTAGCTCCTC AACCAAATCCT-3'; GAPDH forward, 5'-CGACCACTT TGTCAAGCTCA-3' and reverse, 5'-GGTTGAGCACAGGGT ACTTTATT-3'. Relative KIF20B gene expression was calculated using the $2^{-\Delta \Delta \mathrm{Cq}}$ method (11).

RNA interference and transfection. A total of four small hairpin (sh)RNAs were purchased from Vigene Biosciences (Rockville, MA, USA). However, shRNA 1 (NM_016195) was the most 
effective one and was therefore used in subsequent experiments (NM_016195, AATAAATTTCGATGGCATTAAGC). KIF20B was silenced using the following specific short hairpin RNA (shRNA) with sequences. A scrambled sequence was used as a negative control. The shRNAs was synthesized by ViGene Bioscience (SH836784). Lipofectamine ${ }^{\circledR} 3000$ (Invitrogen; Thermo Fisher Scientific, Inc.) was used for the shRNA transfection $(500 \mathrm{ng} / \mu \mathrm{l})$, according to the manufacturer's protocol. A total of $10 \times 10^{5}$ cells per well in a 6 -well plate were provided in four groups including: Sh-KIF20B group transfected with shRNA targeting KIF20B, negative control group transfected with promiscuous sequences without transfection. Silencing efficiency was measured by RT-PCR and western blotting after $48 \mathrm{~h}$ transfection.

Western blotting. A total of $50 \mu \mathrm{g}$ of protein was extracted by protein lysate buffer (cat. no. M329-10ML; VWR International, Radnor, PA, USA) from CAL27 or TCA8113 cells, and the concentration of protein was measured with a bicinchoninic acid protein assay kit (Beyotime Institute of Biotechnology, Haimen, China). Then the protein was transferred to nitrocellulose membranes after 10\% SDS-PAGE. The membranes were blocked and incubated with primary antibodies, including anti-KIF20B antibody (cat. no. NBP1-88042; 1:100; Novus Biologicals), mouse anti- $\beta$-actin (cat. no. 3700; 1:500; CST Biological Reagents Co., Ltd., Shanghai, China), rabbit anti-Ki67 (1:1,000; cat. no. ab16667; Abcam, Cambridge, $\mathrm{UK}$ ), mouse anti-proliferating cell nuclear antigen (PCNA; 1:1,000; cat. no. 2586; CST Biological Reagents Co., Ltd.) monoclonal antibodies overnight at $4^{\circ} \mathrm{C}$, and then incubation with the goat anti-rabbit (1:10,000; cat. no. 711-1122; Rockland Immunochemicals, Inc., Limerick,PA, USA) or goat anti-mouse (1:10,000; cat. no. 110-1103; Rockland Immunochemicals, Inc., Limerick, PA, USA) secondary antibodies at $37^{\circ} \mathrm{C}$ for $1 \mathrm{~h}$. Western blot data were quantified by densitometric analysis with ImageJ software version 1.8.0 (National Institutes of Health, Bethesda, MD, USA), and the relative expression level was normalized by the internal standard $\beta$-actin.

Colony formation assay. Each group of cells in the logarithmic growth phase was selected and digested with $0.25 \%$ trypsin and then splashed into single cells. The cell suspensions were diluted and each group had a gradient density of 50, 100, 200 cells per dish and were inoculated with $10 \mathrm{ml}$ at $37^{\circ} \mathrm{C}$ culture medium in the dish, and cells were shaken gently to be evenly dispersed. The cells were incubated in a $37^{\circ} \mathrm{C}, 5 \% \mathrm{CO}_{2}$ and saturated cell culture incubator for 7 days. When macroscopic colonies appeared in the culture dish, the culture was terminated. The supernatant was discarded and cells were carefully washed twice with PBS. Cells were fixed with $4 \%$ paraformaldehyde at room temperature for $15 \mathrm{~min}$. Subsequently, the fixative was removed, and an appropriate amount of GIMSA staining solution was used at room temperature for 10-30 min. Subsequently, cells were washed with running water and left to dry in the air. Colonies were then counted with a light microscope.

MTT assay for cell viability. Cells were cultured in the aforementioned culture solutions with $10 \%$ fetal bovine serum and 5,000 cells per well were seeded into 96-well plates. After
3-5 days of culture, $20 \mu \mathrm{l}$ of MTT solution was added $(5 \mathrm{mg} / \mathrm{ml}$ in PBS) to each well for $4 \mathrm{~h}$ at $37^{\circ} \mathrm{C}$. The culture supernatant was carefully discarded. Subsequently, cells were centrifuged $560 \mathrm{x} \mathrm{g}$ at $4^{\circ} \mathrm{C}$. A total of $150 \mu \mathrm{l}$ dimethyl sulfoxide was added to each well and was shaken for $10 \mathrm{~min}$ at room temperature to allow the pellet to fully dissolve. Optical density was obtained at a wavelength of $490 \mathrm{~nm}$.

In vivo xenograft assay. BALB/c nude mice (age, 5 weeks; female, 20-25 g; $n=10$ ) were provided by Shanghai SLAC Laboratory Animal Co., Ltd. (Shanghai, China) and mice were housed in pathogen-free animal facilities at $20-25^{\circ} \mathrm{C}$ and $40-70 \%$ humidity in a $12 \mathrm{~h}$ light/dark cycle, with free access to food and water. A total of $2 \times 10^{6}$ cells were injected subcutaneously into nude mice and the tumor volume was measured. Mice (8 per group) were randomized into two groups and treated with CAL-27 control cells and CAL-27 KIF20B shRNA cells. After injection, the animals were housed for three weeks. The volume of the tumor was measured every three days from the third week using a Vernier caliper. The tumor volume was calculated as follows: Tumor volume $\left(\mathrm{mm}^{3}\right)=$ Tumor length $(\mathrm{mm}) \mathrm{x}$ Tumor width $(\mathrm{mm})^{2} / 2$. The present study was approved by the Laboratory Animal Ethics Committee of the Fifth Central Hospital of Tianjin (SYXK 2017-0210).

Statistical analysis. The data were analyzed by IBM SPSS version 22.0 software (IBM Corp., Armonk, NY, USA). For immunohistochemistry experiments, the association between KIF20B expression and clinicopathological features was assessed using the $\chi^{2}$ test. Association of survival and tumor progression with KIF20B expression was estimated by the Kaplan-Meier method and the log-rank test. Data are shown as the mean \pm standard deviation. Student's t-test was used for statistical comparisons. $\mathrm{P}<0.05$ was considered to indicate a statistically significant difference.

\section{Results}

KIF20B is highly expressed in tongue cancer and is associated with poor clinicopathological features. KIF20B antibody was used on the cancer tissue and corresponding paraneoplastic tissue for the immunohistochemistry experiments, and it was found that there was significantly higher expression levels of KIF20B in cancer tissues, compared with the noncancerous ones. Tongue cancer (Fig. 1A) and paired para-carcinoma tissue samples (Fig. 1B) were collected from 82 patients, and were divided into high and low groups according to the expression of KIF20B. High KIF20B expression was found in 40/82 cases and low KIF20B was found in 42/82 cancerous tissues. The results showed that the expression levels of KIF20B were different between carcinoma and adjacent normal tissues. High KIF20B expression was found in 25/82 cases and low expression in 57/82 cases. High KIF20B expression was relatively lower in adjacent normal tissues (high-positive rate, $40 / 82$ vs. $\left.25 / 82 ; \chi^{2}=7.293 ; \mathrm{P}<0.05\right)$. By analyzing the clinicopathological data of the two groups, the expression level of KIF20B was closely associated with clinical stage $(\mathrm{P}<0.05)$ and lymph node metastasis $(\mathrm{P}<0.05)$ in tongue cancer, but not with age, sex, tumor size and cell differentiation (Table I). The differences in tumor differentiation refers to a comparison 
A

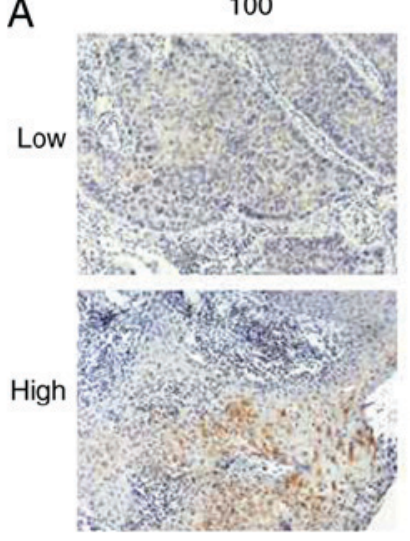

200

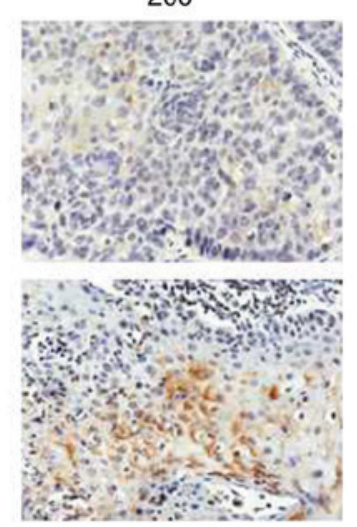

B

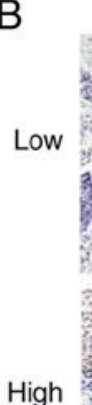

100

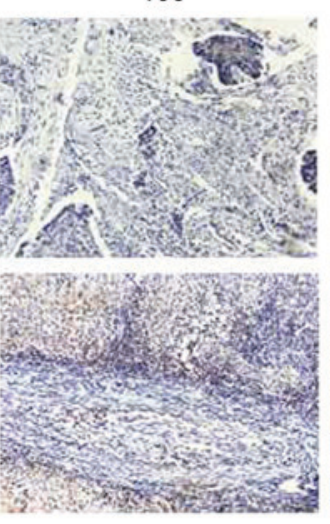

200

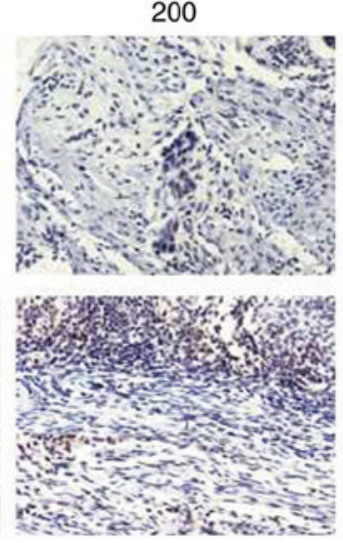

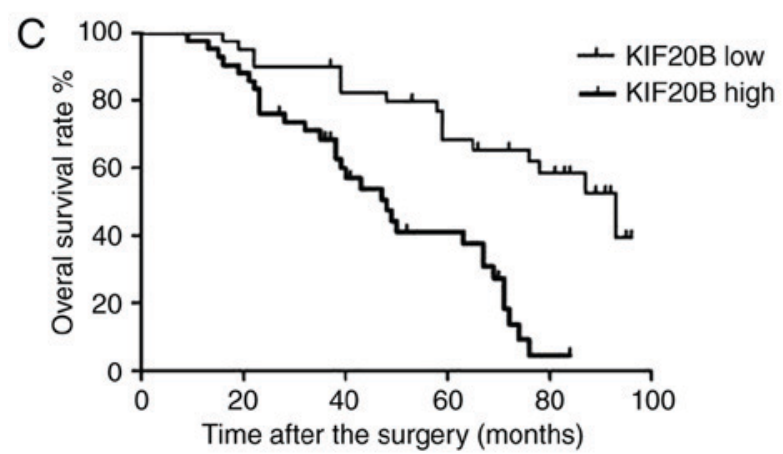

Figure 1. Immunohistochemical analysis of correlation between KIF20B and clinical outcomes of tongue cancer patients. (A) Representative immunohistochemistry images of KIF20B high and low expression groups in the tongue cancer tissues. (B) Representative immunohistochemistry images of KIF20B high and low expression groups in the normal tissue adjacent to carcinoma. (C) The Kaplan-Meier analysis of the survival time after surgery. KIF, kinesin family member.

of well-differentiated and moderately-poorly differentiated tumors. Clinical stage refers to the phase I, II, III and IV comparison, as seen in Table I. From the above results, it seems that patients with high expression of KIF20B had significantly poor clinical and pathological features, compared with the low-expression group.

High KIF20B expression leads to poor clinical outcomes. Kaplan-Meier survival curve was used to analyze the clinical outcomes of these two groups of patients. A 5-year overall survival (OS) and disease-free survival (DFS) was performed with these 82 patients. As shown in Fig. 1C, postoperative OS and DFS for KIF20B high expression group were significantly worse than the low expression group $(\mathrm{P}<0.05)$. It can be seen that the KIF20B high expression group was significantly poorer both in OS and in DFS. These results indicate that the expression of KIF20B could affect the overall postoperative survival time and recurrence-free survival time in patients with tongue cancer, and was closely associated with the clinical outcomes of tongue cancer.

Knockdown of KIF20B suppresses cancer cell proliferation. Based on the above findings, the role of KIF20B in tongue cancer cell lines was investigated. Two tongue cancer cell lines, CAL-27 and TCA8113 were used for the in vitro experiment. The expression of KIF20B in both cell lines was investigated by RT-qPCR and western blotting. KIF20B was knocked down in both cell lines using shRNA and the knockdown efficiency was tested by RT-qPCR and western blotting (Fig. 2). The results showed that KIF20B was expressed in both cell lines and shRNA knocked down the expression of KIF20B in both cell lines successfully.

MTT and colony formation assay were used to detect the impact of KIF20B knockdown in the viability of these two cell lines. Following knockdown of KIF20B, proliferative ability of CAL-27 and TCA8113 cells was significantly inhibited (Fig. 3A and B), indicating that the knockdown of KIF20B might inhibit cell proliferation. Furthermore, expression levels of proliferation-associated proteins PCNA and Ki67 were investigated by western blotting (Fig. 3C and D). The results showed that the expression levels of Ki67 and PCNA were significantly decreased after knockdown of KIF20B $(\mathrm{P}<0.05)$, suggesting that KIF20B may regulate the proliferation of tongue cancer cells by affecting the expression of PCNA and Ki67, but this needs to be validated with further experiments.

Knocking down KIF2OB in vivo results in smaller tumor. Through the above experiments and analysis, it seems that knockdown of KIF20B could inhibit the proliferation of tumor cells in vitro, but it was not known whether it had the similar effect in vivo. Therefore, a subcutaneous tumor formation experiment in nude mice was performed to explore the impact of knocking down KIF20B on tumor formation in vivo. Nude mice were divided randomly into two groups, one was subcutaneously implanted with shRNA-KIF20B cells, which lacked KIF20B expression, and the other group was subcutaneously implanted with normal control cells. 
Table I. Association between KIF20B expression and clinicopathological characteristics in 82 patients with tongue cancer.

\begin{tabular}{|c|c|c|c|c|c|}
\hline \multirow[b]{2}{*}{ Feature } & \multirow[b]{2}{*}{ All n=82 } & \multicolumn{2}{|c|}{ KIF20B expression } & \multirow[b]{2}{*}{$\chi^{2}$} & \multirow[b]{2}{*}{ P-value } \\
\hline & & Low $n=40$ & High $n=42$ & & \\
\hline Age (years) & & & & 0.597 & 0.440 \\
\hline$<65$ & 54 & 28 & 26 & & \\
\hline$\geq 65$ & 28 & 12 & 16 & & \\
\hline Sex & & & & 1.179 & 0.278 \\
\hline Male & 46 & 20 & 26 & & \\
\hline Female & 36 & 20 & 16 & & \\
\hline Clinical stage & & & & 6.057 & $0.014^{\mathrm{a}}$ \\
\hline I-II & 30 & 20 & 10 & & \\
\hline III-IV & 52 & 20 & 32 & & \\
\hline Differentiation & & & & 3.241 & 0.072 \\
\hline Low & 24 & 8 & 16 & & \\
\hline High & 58 & 32 & 26 & & \\
\hline Tumor size & & & & 0.532 & 0.466 \\
\hline$<3$ & 50 & 26 & 24 & & \\
\hline$\geq 3$ & 32 & 14 & 18 & & \\
\hline Lymph node metastasis & & & & 6.129 & $0.013^{\mathrm{a}}$ \\
\hline Yes & 36 & 12 & 24 & & \\
\hline No & 46 & 28 & 18 & & \\
\hline
\end{tabular}

KIF, kinesin family member. ${ }^{\mathrm{a}} \mathrm{P}<0.05$.
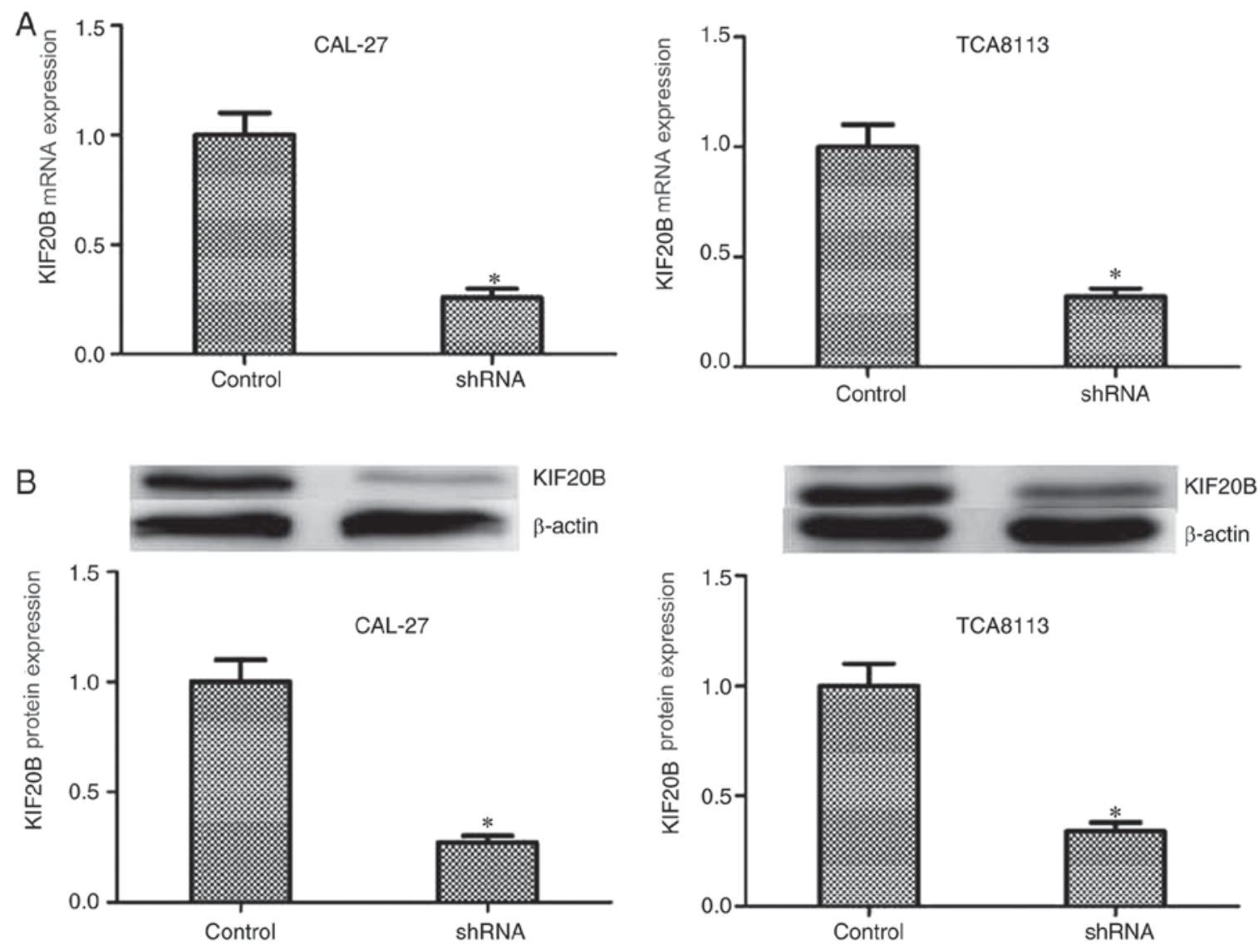

Figure 2. KIF20B shRNA efficiency. (A) The shRNA efficiency measured by reverse transcription-quantitative polymerase chain reaction and (B) by western blotting. ${ }^{~} \mathrm{P}<0.05$ vs. control. KIF, kinesin family member; shRNA, short hairpin RNA. 
A
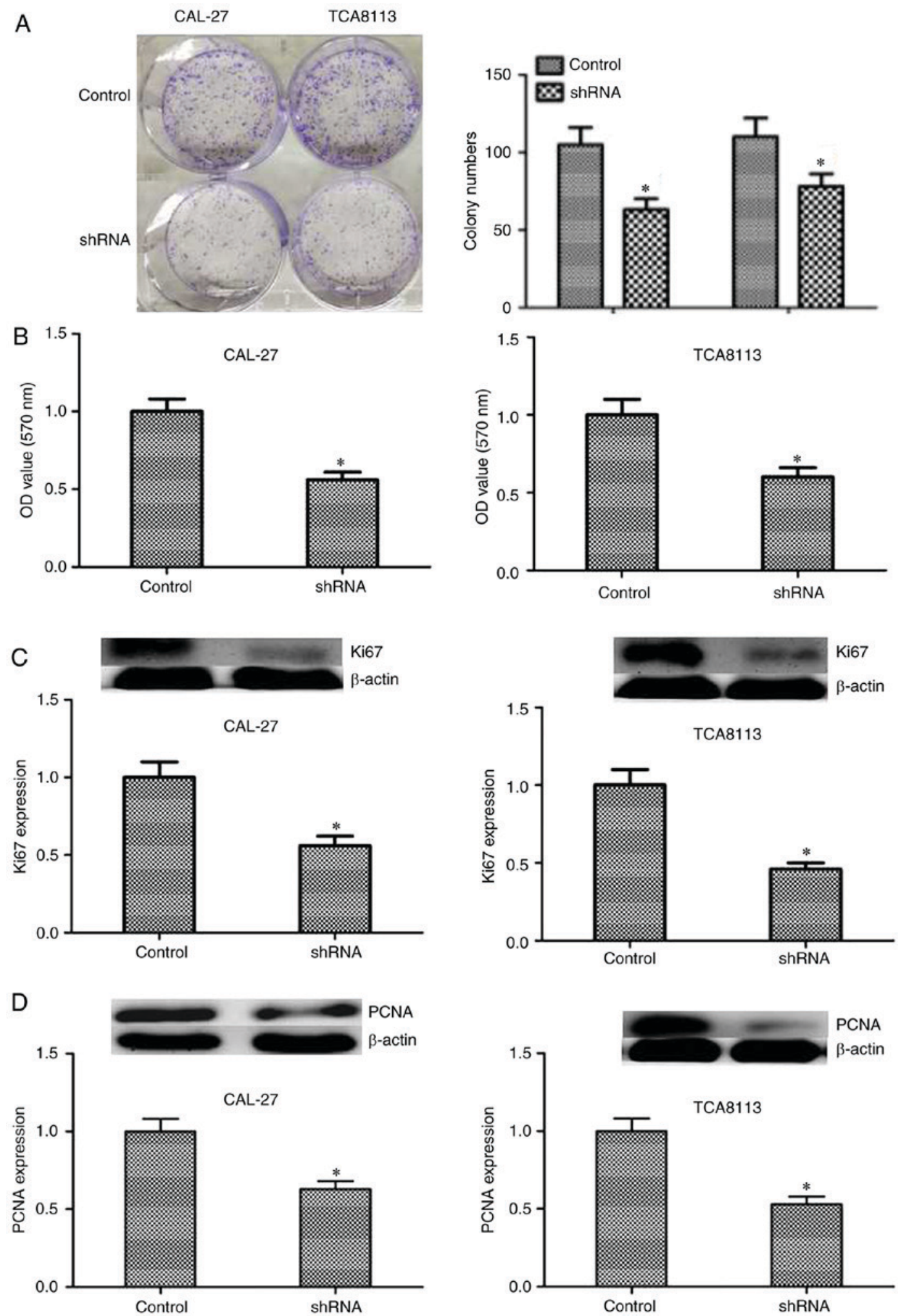

Figure 3. KIF20B knockdown inhibits cell proliferation. (A) Colony formation assay in CAL-27 and TCA-8113 cells. Knocking down KIF20B results in fewer colonies. (B) MTT assay in CAL-27 and TCA8113 cells. (C) Expression of proliferation marker Ki67 and (D) PCNA in shRNA cells and control cells, measured by western Blotting. Knocking down KIF20B inhibited PCNA and Ki67 expression. "P<0.05 vs. control. KIF, kinesin family member; PCNA, proliferating cell nuclear antigen; shRNA, short hairpin RNA.

The volume of the tumors were analyzed after 4 weeks, and the results showed that, the tumor volume of shRNA group was significantly smaller than that of the control $(\mathrm{P}<0.05$; Fig. 4A). KIF20B was indeed significantly knocked down in shRNA group, as detected by western blotting (Fig. 4B) and immunohistochemistry (Fig. 4C). Immunohistochemistry results also showed that Ki67 and PCNA protein expression levels were significantly decreased after knockdown of KIF20B ( $\mathrm{P}<0.05$; Fig. 4D). The above results showed that knocking down KIF20B could reduce tumor growth in vivo. 

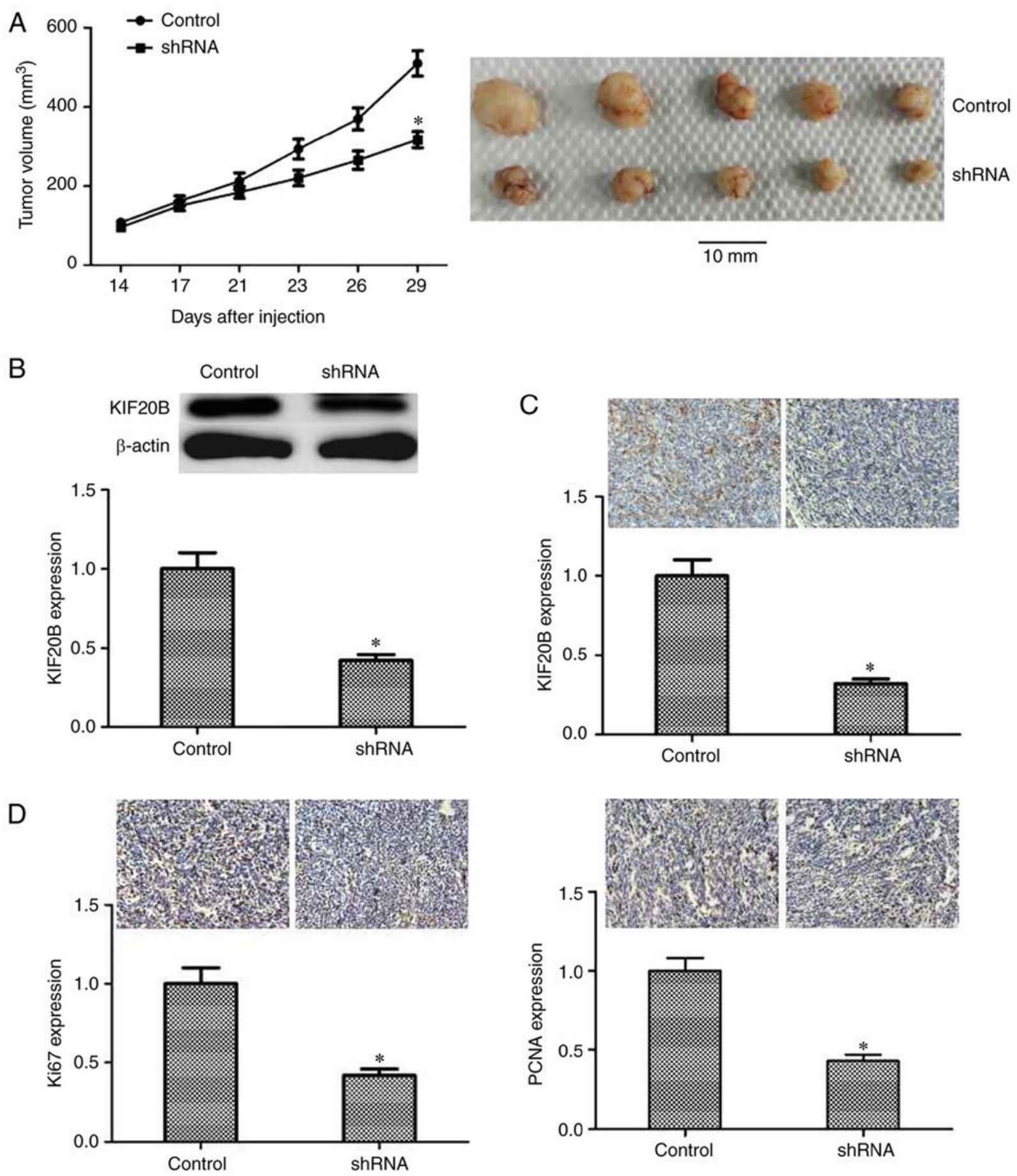

Figure 4. KIF20B knockdown inhibits tumor growth in vivo. (A) Tumor volume in KIF20B knockdown and control nude mice groups after 3 weeks. (B) Effective knockdown efficiency tested by western blotting and (C) immunohistochemistry. (D) Expression of proliferation markers PCNA and Ki67 as measured by immunohistochemistry. "P<0.05 vs. control. KIF, kinesin family member; PCNA, proliferating cell nuclear antigen; shRNA, short hairpin RNA.

\section{Discussion}

KIF20B was first identified as a plus-end-directed kinesin-associated protein and is phosphorylated at the G2/M transition (3). KIF20B could also influence microtubule-binding, microtubule-bundling properties and microtubules-stimulated ATPase activity in vitro, and is essential for cytokinesis in cell cycle (5). KIF20B is reported to be required for efficient cytokinetic furrowing and timely abscission in human cells, which could regulate late steps of maturation including ESCRT-III recruitment and the microtubule constriction sites formation (27). Due to the important function of KIF family, abnormal KIFs expression often leads to a series of cell dysfunction. Overexpression of KIFs might result in a series of errors including excessive spindle separation, premature separation of sister chromatids, advanced leads, and eventually bipolar or unipolar spindles, while lost expression of KIFs might lead to the opposite result (28). Abnormalities in these functions might eventually lead to the development of different diseases, including tumors (3-10). KIF20A was reported to promote the invasiveness of pancreatic cancer by regulating the transport 
of insulin like growth-binding protein 3-containing stress granules (29-32). Downregulation of KIF20A could cause genistein-induced mitotic arrest in gastric cancer cells (33), and KIF20A also had a role in breast cancer (34). Shear variants of KIF23 can lead to shortened survival in patients with hepatocellular carcinoma (35), and high expression of KIF23 is closely associated with the survival of patients with lung cancer (36). KIF23 could also promote glioma progression and being regulated by transcription factor $4(37,38)$. As a member of kinesin-6 family, KIF20B functions as cancer-testis antigen specific to human bladder cancer $(4,5)$, which is associated with cell proliferation and tumor growth in hepatocellular carcinoma through targeting p53, serving a crucial role in cancer (6-10). However, unlike KIF20A, there are few studies on the function and mechanism of KIF20B in tumors (4,6-10), especially in tongue cancer. In the present study, it was found that KIF20B was significantly overexpressed in tongue cancer patients. These results were consistent with previous studies in other types of cancer (4,6-10). Furthermore, high expression of KIF20B was associated with advanced tumor stage, lymph node metastasis and poor prognosis, suggesting that KIF20B may serve an important role in the development process of tongue cancer. Additionally, consistent with previous reports demonstrating high KIF20B expression is associated with poor prognosis in hepatocellular carcinoma (10), the results of the present study showed that high KIF20B expression may be closely associated with worse clinical prognosis of tongue cancer.

Most studies targeting KIF20B remained focused on its normal function $(3,5,27)$. KIF20B was previously demonstrated to function as an 'onco-protein' in lung, hepatocellular, pancreatic, colorectal and bladder cancer (4,6-10). However, there is no study of tongue cancer about KIF20B. Therefore, cell assays were performed using two oral squamous cell carcinoma cell lines CAL-27 and TCA8113. The expression of KIF20B was measured in both cell lines and KIF20B was subsequently knocked down in both cell lines using shRNA. Consistent with previous studies $(4,6-10)$, the results of the present study demonstrated that decreased KIF20B significantly inhibited tumor cell proliferation by mediating proliferation-associated protein expression, such as PCNA and Ki67.

By cell experiments, KIF20B is likely to serve an important role in the proliferation and metastasis of tongue cancer cells. To further verify if KIF20B has similar effects in vivo, a subcutaneous tumorigenic experiment was performed in nude mice using stable knockdown cell lines. The results showed that knockdown of KIF20B could inhibit tumor volume, thereby inhibiting tumor progression, in vivo. Perhaps owing to the small number of the cells and the low malignancy of the tumor, there was no significant difference between the control and the shRNA group on day 14, although it seemed that the tumor was a little smaller in the shRNA group than that in the control group. And the significant difference between the control and the shRNA group on the day 29. Based on the above, it seems that KIF20B serves a very important role both in vivo and in vitro. Therefore, KIF20B can affect tongue cancer progression, but this needs to be verified. tongue cancer is a very complex disease, many factors may lead to its occurrence and development (1). There are not many reports on biomarkers for tongue cancer, so this needs to be investigated further.
KIF20B may be a potential tumor marker for tongue cancer and its expression levels may be able to reflect the progression status of patients with tongue cancer, to some extent, making it a potential factor to predict the prognosis, but this needs to be validated further.

In conclusion, in the present study, KIF20B was found to be closely associated with tumor differentiation, lymph node metastasis and clinical stage through clinical analysis, as well as with prognostic indicators such as survival and recurrence. Subsequently, knockdown of KIF20B could inhibit cell proliferation, in vitro. Additionally, knockdown of KIF20B could reduce tumor volume, in vivo. KIF20B could promote tongue cancer progression in vitro and in vivo, therefore KIF20B might be a potential biomarker for tongue cancer, and might be a novel target for biotherapy.

\section{Acknowledgements}

Not applicable.

\section{Funding}

The present study was supported by Tianjin Binhai new district health planning commission project (Grant no. 2015BWKY003).

\section{Availability of data and materials}

The datasets used and/or analyzed during the current study are available from the corresponding author on reasonable request.

\section{Authors' contributions}

Z-YL,Z-XW and C-CL conceived and designed the experiments. $\mathrm{Z}-\mathrm{YL}$ and Z-XW performed the experiments. C-CL analyzed the data.Z-YL, Z-XW and C-CL contributed reagents, materials and analytical tools. Z-YL and C-CL wrote the manuscript.

\section{Ethics approval and consent to participate}

The present study was approved by the Ethics Committee of the Fifth Central Hospital of Tianjin. Participants provided written informed consent. The study on animals was approved by the Laboratory Animal Ethics Committee of the Fifth Central Hospital of Tianjin (SYXK 2017-0210).

\section{Patient consent for publication}

Not applicable.

\section{Competing interests}

The authors declare that they have no competing interests.

\section{References}

1. Lingen MW, Kalmar JR, Karrison T and Speight PM: Critical evaluation of diagnostic aids for the detection of oral cancer. Oral Oncol 44: 10-22, 2008.

2. Siegel RL, Miller KD and Jemal A: Cancer statistics, 2017. CA Cancer J Clin 67: 7-30, 2017. 
3. Matsumoto-Taniura N, Pirollet F, Monroe R, Gerace L and Westendorf JM: Identification of novel M phase phosphoproteins by expression cloning. Mol Biol Cell 7: 1455-1469, 1996.

4. Kanehira M, Katagiri T, Shimo A, Takata R, Shuin T, Miki T, Fujioka T and Nakamura Y: Oncogenic role of MPHOSPH1, a cancer-testis antigen specific to human bladder cancer. Cancer Res 67: 3276-3285, 2007.

5. Abaza A, Soleilhac JM, Westendorf J, Piel M, Crevel I, Roux A and Pirollet F: M phase phosphoprotein 1 is a human plus-end-directed kinesin-related protein required for cytokinesis. J Biol Chem 278: 27844-27852, 2003.

6. Obara W, Ohsawa R, Kanehira M, Takata R, Tsunoda T, Yoshida K, Takeda K, Katagiri T, Nakamura Y and Fujioka T: Cancer peptide vaccine therapy developed from oncoantigens identified through genome-wide expression profile analysis for bladder cancer. Jpn J Clin Oncol 42: 591-600, 2012.

7. Liu X,Zhou Y, Liu X, Peng A, Gong H, Huang L, Ji K, Petersen RB, Zheng L and Huang K: MPHOSPH1: A potential therapeutic target for hepatocellular carcinoma. Cancer Res 74: 6623-6634, 2014.

8. Lin WF, Lin XL, Fu SW, Yang L, Tang CT, Gao YJ, Chen HY and Ge ZZ: Pseudopod-associated protein KIF20B promotes Gli1-induced epithelial-mesenchymal transition modulated by pseudopodial actin dynamic in human colorectal cancer. Mol Carcinog 57: 911-925, 2018.

9. Ansari D, Andersson R, Bauden MP, Andersson B, Connolly JB, Welinder C, Sasor A and Marko-Varga G: Protein deep sequencing applied to biobank samples from patients with pancreatic cancer. J Cancer Res Clin Oncol 141: 369-380, 2015.

10. Liu X, Li Y, Zhang X, Liu XY, Peng A, Chen Y, Meng L, Chen H, Zhang Y, Miao X, et al: Inhibition of kinesin family member 20B sensitizes hepatocellular carcinoma cell to microtubule-targeting agents by blocking cytokinesis. Cancer Sci 109: 3450-3460, 2018

11. Livak KJ and Schmittgen TD: Analysis of relative gene expression data using real-time quantitative PCR and the 2(-Delta Delta C(T)) method. Methods 25: 402-408, 2001.

12. Miki H, Okada Y and Hirokawa N: Analysis of the kinesin superfamily: Insights into structure and function. Trends Cell Biol 15: 467-476, 2005

13. Schlisio S, Kenchappa RS, Vredeveld LC, George RE, Stewart R, Greulich H, Shahriari K, Nguyen NV, Pigny P, Dahia PL, et al: The kinesin KIF1Bbeta acts downstream from EglN3 to induce apoptosis and is a potential 1p36 tumor suppressor. Genes Dev 22: 884-893, 2008.

14. Hirokawa N: Organelle transport along microtubules-the role of KIFs. Trends Cell Biol 6: 135-141, 1996.

15. Hirokawa N: Kinesin and dynein superfamily proteins and the mechanism of organelle transport. Science 279: 519-526, 1998

16. Hirokawa $\mathrm{N}$ and Takemura R: Molecular motors and mechanisms of directional transport in neurons. Nat Rev Neurosci 6 : 201-214, 2005

17. Tipton AR, Wren JD, Daum JR, Siefert JC and Gorbsky GJ GTSE1 regulates spindle microtubule dynamics to control Aurora B kinase and Kif4A chromokinesin on chromosome arms. J Cell Biol 216: 3117-3132, 2017.

18. Li QR, Yan XM, Guo L, Li J and Zang Y: AMPK regulates anaphase central spindle length by phosphorylation of KIF4A J Mol Cell Biol 10: 2-17, 2018.

19. Gu Y, Lu L, Wu L, Chen H, Zhu W and He Y: Identification of prognostic genes in kidney renal clear cell carcinoma by RNAseq data analysis. Mol Med Rep 15: 1661-1667, 2017

20. Hu CK, Coughlin M, Field CM and Mitchison TJ: KIF4 regulates midzone length during cytokinesis. Curr Biol 21: 815-824, 2011.

21. Lee YM, Lee S, Lee E, Shin H, Hahn H, Choi W and Kim W: Human kinesin superfamily member 4 is dominantly localized in the nuclear matrix and is associated with chromosomes during mitosis. Biochem J 360: 549-556, 2001.

22. Wu G and Chen PL: Structural requirements of chromokinesin Kif4A for its proper function in mitosis. Biochem Biophys Res Commun 372: 454-458, 2008.
23. Wandke C, Barisic M, Sigl R, Rauch V, Wolf F, Amaro AC, Tan CH, Pereira AJ, Kutay U, Maiato H, et al: Human chromokinesins promote chromosome congression and spindle microtubule dynamics during mitosis. J Cell Biol 198: 847-863, 2012.

24. Adams RR, Tavares AA, Salzberg A, Bellen HJ and Glover DM: Pavarotti encodes a kinesin-like protein required to organize the central spindle and contractile ring for cytokinesis. Genes Dev 12: 1483-1494, 1998

25. Hill E, Clarke M and Barr FA: The Rab6-binding kinesin, Rab6-KIFL, is required for cytokinesis. EMBO J 19: 5711-5719, 2000.

26. Nislow C, Lombillo VA, Kuriyama R and McIntosh JR: A plus-end-directed motor enzyme that moves antiparallel microtubules in vitro localizes to the interzone of mitotic spindles. Nature 359: 543-547, 1992.

27. Janisch KM, McNeely KC, Dardick JM, Lim SH and Dwyer ND: Kinesin-6 KIF20B is required for efficient cytokinetic furrowing and timely abscission in human cells. Mol Biol Cell 29: 166-179, 2018.

28. Lucanus AJ and Yip GW: Kinesin superfamily: Roles in breast cancer, patient prognosis and therapeutics. Oncogene 37: 833-838, 2018

29. Taniuchi K, Furihata M and Saibara T: KIF20A-mediated RNA granule transport system promotes the invasiveness of pancreatic cancercells. Neoplasia 16: 1082-1093, 2014

30. Stangel D, Erkan M, Buchholz M, Gress T, Michalski C, Raulefs S, Friess H and Kleeff J: Kif20a inhibition reduces migration and invasion of pancreatic cancer cells. J Surg Res 197: 91-100, 2015.

31. Taniuchi K, Nakagawa H, Nakamura T, Eguchi H, Ohigashi H, Ishikawa O, Katagiri T and Nakamura Y: Down-regulation of RAB6KIFL/KIF20A, a kinesin involved with membrane trafficking of discs large homologue 5, can attenuate growth of pancreatic cancer cell. Cancer Res 65: 105-112, 2005.

32. Asahara S, Takeda K, Yamao K, Maguchi $\mathrm{H}$ and Yamaue $\mathrm{H}$ : Phase I/II clinical trial using HLA-A24-restricted peptide vaccine derived from KIF20A for patients with advanced pancreatic cancer. J Transl Med 11: 291, 2013.

33. Yan GR, Zou FY, Dang BL, Zhang Y, Yu G, Liu X and He QY: Genistein-induced mitotic arrest of gastric cancer cells by downregulating KIF20A, a proteomics study. Proteomics 12: 2391-2399, 2012

34. Khongkow P, Gomes AR, Gong C, Man EP, Tsang JW, Zhao F, Monteiro LJ, Coombes RC, Medema RH, Khoo US and Lam EW: Paclitaxel targets FOXM1 to regulate KIF20A in mitotic catastrophe and breast cancer paclitaxel resistance. Oncogene 35 990-1002, 2016

35. Sun X, Jin Z, Song X, Wang J, Li Y, Qian X, zhang Y and Yin Y: Evaluation of KIF23 variant 1 expression and relevance as a novel prognostic factor in patients with hepatocellular carcinoma. BMC Cancer 15: 961,2015.

36. Kato T, Wada H, Patel P, Hu HP, Lee D, Ujiie H, Hirohashi K, Nakajima T, Sato M, Kaji M, et al: Overexpression of KIF23 predicts clinical outcome in primary lung cancer patients. Lung Cancer 92: 53-61, 2016

37. Takahashi S, Fusaki N, Ohta S, Iwahori Y, Iizuka Y, Inagawa K, Kawakami Y, Yoshida K and Toda M: Downregulation of KIF23 suppresses glioma proliferation. J Neurooncol 106: 519-529, 2012.

38. Sun L, Zhang C, Yang Z, Wu Y, Wang H, Bao Z and Jiang T: KIF23 is an independent prognostic biomarker in glioma, transcriptionally regulated by TCF-4. Oncotarget 7: 24646-24655, 2016.

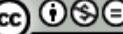

This work is licensed under a Creative Commons Attribution-NonCommercial-NoDerivatives 4.0 International (CC BY-NC-ND 4.0) License. 